\title{
Welche Personenmerkmale sagen Medienvertrauen voraus?
}

\section{Der Einfluss von Charakteristika der Rezipientinnen und Rezipienten auf Vertrauen in Medien im Zeitverlauf}

\author{
Ilka Jakobs • Nikolaus Jackob • Tanjev Schultz • Marc Ziegele • \\ Christian Schemer • Oliver Quiring
}

Eingegangen: 8. Januar 2021 / Angenommen: 11. April 2021 / Online publiziert: 28. Juni 2021

(C) Der/die Autor(en) 2021

Zusammenfassung In den letzten Jahren haben viele Studien untersucht, welche Faktoren das Vertrauen in Medien beeinflussen können. Zu einigen dieser Faktoren gibt es vorläufige wissenschaftliche Erkenntnisse, die eine klare Richtung andeuten, $\mathrm{zu}$ anderen ist die Forschungslage eher heterogen. Wir überprüfen daher anhand einer Trendstudie mit Befragungsdaten aus den Jahren 2017, 2018 und 2019 mit je 1200 Befragten, welche Personenmerkmale sich in Deutschland über einen längeren Zeitraum als stabile Einflussfaktoren erwiesen haben. Dazu führen wir blockweise Regressionsanalysen im Zeitverlauf durch. Neben soziodemografischen Faktoren und der Mediennutzung gehören dazu die Einstellungen zu Politik, Verschwörungsglaube und interpersonales Vertrauen. Die Ergebnisse zeigen, dass alle Gruppen von Variablen mit Medienvertrauen im Zusammenhang stehen, dass ihr Einfluss jedoch unterschiedlich groß ist. In komplexeren Regressionsmodellen wird der Einfluss

\footnotetext{
Dr. Ilka Jakobs $(\varangle) \cdot$ PD Dr. Nikolaus Jackob · Prof. Dr. Tanjev Schultz · Prof. Dr. Christian Schemer · Prof. Dr. Oliver Quiring

Institut für Publizistik, Johannes Gutenberg-Universität Mainz, Jakob-Welder-Weg 12, 55099 Mainz, Deutschland

E-Mail: ilka.jakobs@uni-mainz.de

PD Dr. Nikolaus Jackob

E-Mail: nikolaus.jackob@uni-mainz.de

Prof. Dr. Tanjev Schultz

E-Mail: tanjev.schultz@uni-mainz.de

Prof. Dr. Christian Schemer

E-Mail: schemer@uni-mainz.de

Prof. Dr. Oliver Quiring

E-Mail: quiring@uni-mainz.de

Jun.-Prof. Dr. Marc Ziegele

Institut für Sozialwissenschaften, Heinrich-Heine-Universität Düsseldorf,

Ulenbergstraße 127, 40225 Düsseldorf, Deutschland

E-Mail: marc.ziegele@hhu.de
} 
mancher Variablen vom Effekt anderer Variablen überlagert - dies könnte eine Erklärung für die bislang äußerst heterogene Forschungslage zu beispielsweise dem Einfluss der Soziodemografika auf Medienvertrauen sein. Die Ergebnisse verdeutlichen auch, welche Rezipientinnen und Rezipienten den Medien weniger oder gar nicht vertrauen.

Schlüsselwörter Medienvertrauen · Einflussfaktoren für Medienvertrauen · Personenmerkmale $\cdot$ Regressionsanalysen $\cdot$ Langzeitstudie

\section{How stable are predictions of media trust based on recipients' characteristics?}

On the effect of recipient's characteristics on media trust in a long-term perspective

Abstract Media trust has become a highly relevant topic in Communication Science in the past years. Trust in societal institutions, e.g., the media, is an important prerequisite for the functioning of democratic systems. The actual debate on the 'lying press' and 'Fake News' has intensified research on many different aspects of media trust. This encompasses (1) studies on the amount of media trust citizens have and on (2) different forms of media trust. Furthermore, (3) consequences of media trust have been investigated, e.g. how media trust shapes political judgements or perceptions, as well as (4) the media coverage on trust. Other researchers (5) put their focus on methodological aspects and try to improve the operationalization and the measurement of media trust. In addition, (6) factors which can influence media trust have been analyzed-amongst them recipients' characteristics and media use, but, especially in international comparisons, media system factors, societal influence, a country's economic performance or political conditions. The focus of our study is on the influence of recipient's characteristics on media trust. Regarding some factors, as for example sociodemographic variables, their influence is still unclear because of very heterogenous findings. However, regarding other factors, as for example political attitudes, we already know that they seem to influence media trust-but in different studies, this influence is differently intensive. In our study, we want thus to find out which characteristics of recipients including their media use have a stable influence on media trust over time.

Therefore, we use data from a long-term study on media trust: telephone surveys representative for the German population aged 18 and above in 2017, 2018 and 2019 with 1200 participants each. Besides media trust, we asked for different characteristics of respondents that can influence media trust: sociodemographic variables, media use, political attitudes, conspiracy beliefs and interpersonal trust. We use stepwise regression analysis in each year to find out which variables show a stable influence on media trust over time and which amount of media trust can be explained by different kinds of variables.

The results show that all kinds of variables investigated-sociodemographic variables, media use, political attitudes, conspiracy beliefs and interpersonal trust-influenced media trust in the years 2017-2019. In the most complex models, $25 \%$ of the variance in media trust could be explained by these variables. Amongst the sociodemographic variables, age, formal education and gender showed an effect 
on media trust - older people, woman and those with a lower formal education had lower media trust. However, the influence of these variables disappeared partly in more complex regression models. Regarding media use, especially the use of public broadcasting TV and of so-called "alternative" news websites like Russia Today showed a stable effect on media trust-frequent users of public service broadcasting TV had higher, frequent users of alternative news websites lower media trust. The influence of some media types, e.g. newspapers or private TV, disappeared in more complex regression analysis. Political attitudes also were an important factor influencing media trust. Strongest was the effect of satisfaction with the democratic system-those whose satisfaction with our democratic system was high also had a higher media trust. Those whose political apathy was high showed lower levels of media trust. Furthermore, belief in conspiracy theories and interpersonal trust were related to media trust. Those who tended to believe conspiracy theories to be true had lower media trust - those with a high interpersonal trust had higher media trust.

In an additional regression analysis, we analyzed in the data set of the year 2018 other variables which we only had explored in this year. The goal of this additional analysis was to show which kinds of variables also can help to explain media trust and on which variables future research could thus concentrate. The analysis showed that also citizen's personal economic situation, alienation from the media, trust in other societal institutions (e.g., politics, justice) and the willingness for political participation were related to media trust, whereas knowledge about media had no effect on media trust.

Overall, our analysis showed which characteristics of respondents showed a constant influence on media trust over time and whose influence was rather unstable. The effects we found were, due to our long-term study design, independent from the time of measurement, the actual mood in society or current discussions about single issues. We give thus genuine insights in an area where until now, research findings from surveys were rather inconsistent. Despite not analyzing panel data in our study and using a global measurement of media trust, we showed which factors influencing media trust future research could concentrate on and why long-term studies are highly relevant in this field.

Keywords Media Trust · Factors Influencing Media Trust $\cdot$ Recipient's Characteristics · Regression Analysis · Long-Term Study

\section{Einleitung}

Medienvertrauen gewinnt in der kommunikationswissenschaftlichen Forschung der letzten Jahre zunehmend an Bedeutung. Grundlegendes Vertrauen in Institutionen, unter anderem in die Medien, ist eine wichtige Voraussetzung für das Funktionieren der Demokratie (vgl. Dautrich und Hartley 1999, S. 15). Die hartnäckige gesellschaftliche Debatte über „Lügenpresse“ und „Fake News“ (vgl. Engelke et al. 2019, S. 66) hat dem Thema in der Gesellschaft und in der (Kommunikations-)Wissenschaft neue Dringlichkeit verschafft und es zurück auf die Agenda befördert (vgl. Hagen 2015, S. 152). 
Die kommunikationswissenschaftliche Vertrauensforschung widmet sich theoretisch und empirisch diversen Aspekten der Thematik: Erstens gibt es Untersuchungen, die erheben, wie es um das Vertrauen der Menschen in die Medien bestellt ist (vgl. für Deutschland Blöbaum 2018; Schultz et al. 2020; Jakobs et al. 2021; Reinemann et al. 2017; im internationalen Vergleich Hanitzsch et al. 2018; Tsfati und Ariely 2014). Zweitens grenzt die Forschung unterschiedliche Formen von Vertrauen bzw. theoretische Konstrukte voneinander ab (vgl. Strömbäck et al. 2020; Ognyanova 2019). Neben Medienskepsis (vgl. Tsfati und Capella 2003), Medienzynismus (vgl. Jackob et al. 2019a), Medienfeindlichkeit (vgl. Fawzi 2019) und Misstrauen (vgl. Engelke et al. 2019) sind dies u. a. Vertrauen in die Nachrichtenauswahl (vgl. Kohring 2004; Pingree et al. 2013) und Vertrauen in Medien als Institutionen (vgl. Jackob 2012; Jackob et al. 2019b). Drittens stehen die Folgen von Medienvertrauen im Mittelpunkt, wenn beispielsweise politische Entscheidungsfindung, Vertrauen in Institutionen und die Rolle von Medienvertrauen in Agenda-Setting-Prozessen oder bei der Wahrnehmung von Meinungsklima erforscht werden (vgl. Peifer 2018; Tsfati 2003a, 2003b). Viertens geben Analysen der Medienberichterstattung Aufschluss darüber, wie der mediale Diskurs über das Thema Vertrauen gestaltet ist (vgl. Denner und Peter 2017). Fünftens erarbeitet die Forschung in methodischer Hinsicht Verbesserungen für die Operationalisierung und Messung von Medienvertrauen (vgl. Daniller et al. 2017; Engelke et al. 2019; Prochazka und Schweiger 2019). Und schließlich beschäftigt die Forschung sechstens die Frage, welche Faktoren Medienvertrauen beeinflussen können. Neben Merkmalen der Rezipientinnen und Rezipienten inklusive der Mediennutzung werden hier, vor allem international vergleichend, auch Mediensystem-Faktoren, gesellschaftliche Einflüsse, wirtschaftliche Leistung oder politische Gegebenheiten untersucht (vgl. Hanitzsch et al. 2018; Tsfati und Ariely 2014; Yamamoto et al. 2016).

Der sechste und letzte Aspekt steht im Mittelpunkt dieses Beitrages: Mithilfe von drei Befragungswellen einer Langzeitstudie zum Medienvertrauen möchten wir der Frage nachgehen, welche Personenmerkmale nachweislich über einen längeren Zeitraum Einfluss auf das Medienvertrauen der Deutschen nehmen und welche nicht. Die anderen oben angesprochenen Arten von Einflussfaktoren, die sich z. B. auf das Mediensystem beziehen, lassen sich mit unserer Befragungsstudie nicht untersuchen.

Bisherige Studien dazu haben meist nur in einmaligen Befragungen mögliche Einflussfaktoren untersucht - zudem unterscheiden sich die erfassten Faktoren und die Art der Erhebung von Studie zu Studie. Dies hat recht widersprüchliche Forschungsergebnisse zur Folge. Indem wir die Ergebnisse von drei Querschnitts-Befragungen aus den Jahren 2017, 2018 und 2019 heranziehen, in denen das Medienvertrauen erfasst und zudem eine große Zahl an möglichen Einflussfaktoren erhoben wurde, können wir mehrere Messzeitpunkte vergleichen und so Aussagen über die Stabilität des Einflusses von Personenmerkmalen im Zeitverlauf machen. Bei einmaligen Befragungen können beispielsweise die aktuelle gesellschaftliche Stimmung, derzeitige Themenprävalenz oder der Messzeitpunkt den Zusammenhang zwischen Medienvertrauen und Merkmalen der Rezipientinnen und Rezipienten beeinflussen. Da die untersuchten Konstrukte in unseren Befragungswellen stets auf die gleiche Art und Weise erhoben wurden, können wir Zusammenhänge aufzeigen, die über die Zeit 
stabil sind und unabhängig von einzelnen Messzeitpunkten oder Mess-Artefakten zustande kamen.

$\mathrm{Zu}$ wissen, welche Faktoren Medienvertrauen im Zeitverlauf dauerhaft beeinflussen, ist aus mehreren Gründen wichtig: Erstens trägt es zur Beantwortung der Frage bei, welche Menschen den Medien vertrauen und welche nicht - diese Frage spielt in der aktuellen gesellschaftlichen Debatte eine wichtige Rolle. Zweitens kann es ein Ansatzpunkt für die Politik oder die Medien sein, wenn diese versuchen, das Vertrauen der Rezipientinnen und Rezipienten zu steigern, da sie dann wissen, welche Personengruppen sie dafür ansprechen müssen. Drittens leisten wir mit unserer Studie einen Beitrag dazu, das unscharfe Bild, das die kommunikationswissenschaftliche, meist querschnittliche Vertrauensforschung bisher von Einflüssen auf Medienvertrauen hat, durch längsschnittliche Analysen zu schärfen. Und viertens hilft es der Forschung dabei, in künftigen Studien zu entscheiden, welche Kontrollvariablen in Befragungen zu Medienvertrauen erhoben werden sollten.

\section{Medienvertrauen}

Vertrauen wird als eine Beziehung zwischen zwei Akteuren verstanden: der Person, die Vertrauen gibt, und der, die Vertrauen nimmt (vgl. Tsfati und Capella 2003, S. 505). Gambetta beschreibt die Vertrauensbeziehung wie folgt:

When we say that we trust someone or that someone is trustworthy, we implicitly mean that the probability that he will perform an action that is beneficial or at least not detrimental to us is high enough for us to consider engaging in some form of cooperation with him. (Gambetta 1988, S. 217)

Vertrauen reduziert soziale Komplexität (vgl. Luhmann 1989, S. 51; Sztompka 1999, S. 25), beinhaltet für Vertrauensnehmende jedoch immer ein Risiko (vgl. Jackob 2012, S. 96-97): Es ist möglich, dass Vertrauensgebende entgegen den Erwartungen der Vertrauensnehmenden handeln werden (vgl. Tsfati und Capella 2005, S. 252).

Medienvertrauen wird definiert als die Beziehung zwischen den Medien als Vertrauensgebenden und den Rezipientinnen und Rezipienten als Vertrauensnehmenden (vgl. Jackob 2012, S. 96). ${ }^{1}$ Die Menschen vertrauen darauf, dass die Medien über Geschehnisse berichten, die für sie relevant sind und die sie nicht durch eigene Erfahrungen erleben (vgl. Kohring 2004, S. 165). Die Rezipientinnen und Rezipienten sind bei den meisten Themen darauf angewiesen, der Medienberichterstattung auch inhaltlich zu vertrauen (vgl. Cuvalo 2013, S. 149), denn in der Regel sind sie nicht dazu in der Lage oder müssten großen Aufwand treiben, um Informationen aus den Medien anhand von Extra-Media-Daten zu überprüfen (vgl. Tsfati und Peri 2006, S. 171).

\footnotetext{
1 Dabei gilt auch, dass Medien bzw. Journalistinnen und Journalisten in manchen Situationen selbst Vertrauensnehmende sind, wenn sie z. B. bei der Recherche auf die Zuverlässigkeit bestimmter Quellen vertrauen. Auch gegenüber den Rezipientinnen und Rezipienten sind sie in gewisser Weise Vertrauensnehmende - indem sie davon ausgehen, dass diese die Medienprodukte nutzen und damit zur Existenzberechtigung der Medien bzw. deren Wirtschaftlichkeit beitragen.
} 
In der kommunikationswissenschaftlichen Vertrauensforschung hat sich mittlerweile die Erkenntnis durchgesetzt, dass es ,das Medienvertrauen“ nicht gibt, sondern unterschiedliche Formen von Medienvertrauen koexistieren. Das Vertrauen der Menschen kann sich auf unterschiedliche Bezugsobjekte richten: Einerseits kann das Mediensystem als Ganzes Vertrauensobjekt sein, das Vertrauen richtet sich dann auf die Medien als gesellschaftliche Institution (vgl. Jackob 2012, S. 116; Strömbäck et al. 2020, S. 147). Andererseits können Mediengattungen, einzelne Medienmarken, Journalisten und Medieninhalte Bezugsobjekte von Medienvertrauen sein (vgl. Blöbaum 2016, S. 9; Ognyanova 2019, S. 540; Strömbäck et al. 2020, S. 147), wie auch die Selektion und Aufbereitung von Informationen durch Journalistinnen und Journalisten (vgl. Kohring 2004). Mit den unterschiedlichen Vertrauensformen gehen verschiedene Varianten der Messung von Vertrauen einher (siehe ausführlich z. B. Strömbäck et al. 2020, S. 143-145; Engelke et al. 2019; Prochazka und Schweiger 2019, S. 28): Je nachdem, welche Vertrauensform untersucht wird, kommen entweder allgemeine oder sehr spezifische Messungen von Medienvertrauen zum Einsatz.

Mit dem Aufkommen der „Lügenpresse“-Debatte im Jahr 2014 (vgl. Hagen 2015, S. 152) entstanden zunächst Befürchtungen, dass diese Debatte einen Rückgang des Vertrauens der Deutschen in die Medien bewirken könnte (vgl. Reinemann et al. 2017, S. 77-78). In den letzten Jahren stellte sich heraus, dass das nicht unbedingt der Fall ist: „Ein dramatischer Vertrauensverlust über die gesamte Gesellschaft hinweg lässt sich mit seriösen Studien schlicht nicht belegen“, konstatieren Reinemann et al. (2017, S. 91). Blöbaum (2018, S. 601) fand heraus, dass eine ,hartnäckige Medienskepsis [...] nur bei einem kleinen Teil der Bevölkerung sowie insbesondere bei polarisierenden Themen (z. B. Flüchtlingsdebatte)“ vorhanden ist. Auch die Ergebnisse unserer Mainzer Langzeitstudie Medienvertrauen zeigten für das Jahr 2019, dass das „Medienvertrauen in Deutschland vergleichsweise stabil ist“" (Schultz et al. 2020, S. 322), auch wenn wir einen Kern an scharfen Medienkritikerinnen und Medienkritikern ausmachen konnten. In unserer Befragung aus dem Herbst 2019 gaben $43 \%$ der Deutschen an, den Medien bei wichtigen Dingen eher/voll und ganz zu vertrauen. $29 \%$ waren unentschieden, differenzierten also möglicherweise je nach Medienangebot, und $28 \%$ gaben an, den Medien eher nicht/überhaupt nicht zu vertrauen. Im Vergleich zwischen den Mediengattungen zeigten sich dann in der Tat große Unterschiede - der öffentlich-rechtliche Rundfunk genoss mit $67 \%$ die höchsten Vertrauenswerte, gefolgt von Regionalzeitungen (65\%) und überregionalen Tageszeitungen (55\%). Dem privaten Fernsehen (26\%) und Boulevardzeitungen $(7 \%)$ wurde ein wesentlich geringeres Vertrauen zugesprochen. In unserer Befragung im November und Dezember 2020 zeigten sich coronabedingt deutliche Zugewinne an Medienvertrauen. Zynische Sichtweisen wurden tendenziell seltener zu Protokoll gegeben als in den Vorjahren - inwieweit das gestiegene Vertrauen nach der Corona-Pandemie Bestand haben wird, muss sich jedoch erst noch erweisen (vgl. Jakobs et al. 2021). 


\section{Der Einfluss von Personenmerkmalen auf Medienvertrauen}

Zum Einfluss von Personenmerkmalen auf das Medienvertrauen liegt schon viel Forschung vor (vgl. Bentele 2008; Blöbaum 2016; Hanitzsch et al. 2018; Jackob 2012; Jakobs 2018; Jones 2004; Kohring 2004; Lee 2010; Schielicke et al. 2014; Schranz et al. 2016; Schultz et al. 2020; Tsfati und Capella 2003, 2005; Tsfati und Ariely 2014; Yamamoto et al. 2016). Ziel von Studien dieser Art ist es, herauszufinden, welche Rezipientinnen und Rezipienten den Medien in welchem Ausmaß vertrauen und welche das nicht oder in geringerem Ausmaß tun. Zu den Merkmalen, die in der bisherigen Forschung untersucht wurden, gehören die soziodemografischen Variablen, die Mediennutzung, Einstellungen zu Politik, die individuelle Vertrauensbereitschaft und das Vertrauen in andere Institutionen.

Gut erforscht ist der Einfluss von soziodemografischen Merkmalen - jedoch mit widersprüchlichen Ergebnissen: In einigen Studien beeinflussen Geschlecht, Alter oder Bildung das Vertrauen in die Medien (vgl. Schranz et al. 2016; Tsfati und Ariely 2014), in anderen können solche Zusammenhänge nicht nachgewiesen werden (vgl. Jackob 2012; Schielicke et al. 2014). Bei den Studien, die einen Zusammenhang zwischen Soziodemografika und Medienvertrauen finden, haben mal Frauen, mal Männer ein niedrigeres Medienvertrauen, in einigen Studien sind es ältere Befragte, in anderen jüngere (vgl. Schranz et al. 2016, S. 4). Ähnliches zeigt sich bei den Einflüssen der Bildung (vgl. Tsfati und Ariely 2014, S. 762). In der bisherigen Forschung bleibt dabei unklar, welche Gründe diese inkonsistenten Befunde haben. Sie könnten u. a. in den verschiedenen Strukturen der Mediensysteme und in kulturellen Unterschieden zu finden sein, wie z. B. Hanitzsch et al. (2018) anhand des Vergleichs angelsächsisch geprägter und zentraleuropäischer Demokratien vermuten.

Die Mediennutzung gilt als zentrale Einflussgröße (vgl. Schielicke et al. 2014; Donsbach et al. 2009; Jackob 2012; Hopmann et al. 2015; Schranz et al. 2016; Tsfati und Capella 2003; Tsfati und Ariely 2014). Dies gilt unabhängig davon, welche Art von Mediennutzung in Vertrauensstudien untersucht wird: die Nutzung bestimmter Einzelmedien, die Nutzung von Mainstream-Medien oder alternativen Informationsangeboten, die Quellen politischer Informationen oder die Nachrichtennutzung im Internet. Unter den Medien-Faktoren spielt die Glaubwürdigkeit der Medieninhalte eine besonders wichtige Rolle. Glaubwürdigkeit und Vertrauen hängen eng miteinander zusammen; Glaubwürdigkeit kann ein Grund dafür sein, dass Menschen den Medien Vertrauen entgegenbringen. Werden Medieninhalte als unglaubwürdig wahrgenommen, ist es unwahrscheinlicher, dass die Rezipientinnen und Rezipienten den Medien vertrauen (vgl. Jackob 2012; Tsfati und Ariely 2014). Auch Medienmarken und Medienimages spielen eine Rolle: Medienmarken sind Vertrauensanker (vgl. Silberer 2001, S. 237), ein positives Image führt zu einer hohen Akzeptanz eines Mediums (vgl. Siegert 2001, S. 134), was sich auch empirisch in einem höheren Vertrauen niederschlägt (vgl. Jackob 2012, S. 188-192; Jakobs 2018). Jüngst wird auch der Glaube an Verschwörungsideologien als mögliche Erklärung von fehlendem Medienvertrauen herangezogen: In unserer Langzeitstudie Medienvertrauen fanden wir heraus, dass es einen relevanten Kern an Kritikerinnen und Kritikern gibt, die den Medien gegenüber zynisch eingestellt sind, diese pauschal verurteilen und ihnen z.B. vorwerfen, die Bevölkerung systematisch zu belügen oder mit der 
Politik unter einer Decke zu stecken. Wir fanden außerdem einen Zusammenhang zwischen Medienvertrauen und Verschwörungsglauben (vgl. Jackob et al. 2019b).

Bei den Einstellungen zu Politik wurden unterschiedliche Faktoren untersucht, z. B. politisches Interesse, aber auch die Zufriedenheit mit Politik oder Demokratie. Das politische Interesse spielt bei den Vertrauenszuschreibungen an Medien nachgewiesenermaßen eine wichtige Rolle: Je stärker sich die Befragten für Politik interessieren, desto wahrscheinlicher ist es, dass sie den Medien vertrauen (vgl. Schielicke et al. 2014; Schranz et al. 2016; Tsfati und Ariely 2014). Menschen, die die Medien scharf kritisieren (Medienzyniker), zeichneten sich unter anderem durch eine hohe Politikverdrossenheit aus (vgl. Jackob et al. 2019b, S. 215-216). Der Einfluss der Einstellungen zu Politik zeigt sich jedoch nicht in allen Ländern und weist zum Teil ebenfalls in unterschiedliche Richtungen (vgl. Hanitzsch et al. 2018; Lee 2010; Tsfati und Ariely 2014). Auch hier bleiben in der bisherigen Forschung die Gründe für diese inkonsistenten Befunde offen. Das Informationsbedürfnis der Menschen (vgl. Tsfati und Capella 2005; Jackob 2012) sowie ihr Involvement, also der Grad an persönlicher Betroffenheit (vgl. Yamamoto et al. 2016; Jackob 2012), stehen im Zusammenhang mit Vertrauen.

Ein weiterer Block an Einflussfaktoren, die empirisch untersucht wurden, bezieht sich auf die individuelle Vertrauensbereitschaft eines Menschen bzw. das Ausmaß seines Vertrauens in andere Personen oder Institutionen. Ein bestätigter Einflussfaktor ist die individuelle Vertrauenstendenz, also die generelle Bereitschaft einer Person, anderen zu vertrauen. Ist diese hoch, steigt damit auch die Wahrscheinlichkeit, dass den Medien Vertrauen entgegengebracht wird (vgl. Jackob 2012; Tsfati und Ariely 2014). Andere Studien untersuchen das interpersonale Vertrauen als Einflussfaktor: Sie zeigen, dass ein hohes interpersonales Vertrauen zu einem größeren Medienvertrauen führt (vgl. Granow et al. 2020; Tsfati und Ariely 2014). Vertrauen in politische und gesellschaftliche Institutionen hängt ebenfalls mit Medienvertrauen zusammen (vgl. Hanitzsch et al. 2018; Jackob 2012; Jackob et al. 2017) - je höher dieses Vertrauen ist, desto größer ist auch das Medienvertrauen.

Auf Grundlage der vorliegenden Forschung zum Einfluss der Merkmale der Rezipienten auf Medienvertrauen stellen wir folgende Forschungsfragen, die anhand von Befragungsdaten unserer Querschnittsbefragungen aus den Jahren 2017 bis 2019 untersucht werden:

\section{Soziodemografische Faktoren}

F1 Inwieweit beeinflussen Alter, Geschlecht und Bildung das Medienvertrauen?

In Bezug auf die Faktoren Alter, Geschlecht und Bildung ist die Forschungslage sehr heterogen, sodass wir untersuchen, welchen Einfluss diese Faktoren auf das Medienvertrauen im Zeitverlauf zeigen.

\section{Mediennutzung}

F2 Wie beeinflusst die Nutzung unterschiedlicher Mediengattungen das Medienvertrauen? 
Die Mediennutzung hat sich als starker Einflussfaktor herausgestellt - allerdings messen sie verschiedene Studien sehr unterschiedlich. Daher gibt es zwar in der Forschung deutliche Hinweise auf einen Einfluss der Mediennutzung auf das Medienvertrauen. Es ist jedoch unklar, welche Arten der Mediennutzung in welchem Zusammenhang mit Medienvertrauen stehen. Denkbar ist, dass sich die Nutzung verschiedener Mediengattungen unterschiedlich auf Medienvertrauen auswirkt - die Nutzung des öffentlich-rechtlichen Rundfunks sollte andere Auswirkungen auf das Medienvertrauen haben als beispielsweise die Nutzung alternativer Informationsangebote.

\section{Einstellungen zu Politik}

F3 Inwieweit stehen die Einstellungen zu Politik in Form von politischem Interesse, Politikverdrossenheit und Demokratiezufriedenheit im Zusammenhang mit Medienvertrauen?

Die Einstellungen zu Politik haben sich als starker Einflussfaktor herausgestellt. Allerdings wurden in verschiedenen Studien, wie bei der Mediennutzung auch, unterschiedliche Messungen für eine große Zahl an Variablen eingesetzt, mit denen sich die Einstellungen zur Politik erheben lassen. Daher bleibt unklar, welche Aspekte von Einstellungen zu Politik mit Medienvertrauen im Zusammenhang stehen. Dass sich Zusammenhänge zeigen werden, halten wir jedoch für erwartbar.

\section{Verschwörungsglaube}

F4 Wie beeinflusst Verschwörungsglaube das Medienvertrauen?

Erste empirische Ergebnisse zeigen, dass der Verschwörungsglaube mit einem niedrigeren Medienvertrauen einhergeht. Daher untersuchen wir, ob sich dieser Effekt auch im Zeitverlauf zeigt.

\section{Interpersonales Vertrauen}

F5 Inwieweit steht interpersonales Vertrauen im Zusammenhang mit Medienvertrauen?

Einige empirische Befunde zeigen, dass ein niedriges interpersonales Vertrauen mit einem geringeren Medienvertrauen einhergeht. Daher untersuchen wir, ob sich dieser Effekt auch im Zeitverlauf zeigt.

\section{Methode}

In den Jahren 2017, 2018 und 2019 haben wir jeweils im Herbst eine standardisierte, für die deutsche Bevölkerung ab 18 Jahre repräsentative CATI-Befragung durchgeführt. Die Datenerhebungen in den Jahren 2017 und 2018 haben der Impulsfonds der Forschungsinitiative des Ministeriums für Wissenschaft, Weiterbildung und Kultur Rheinland-Pfalz und der Forschungsschwerpunkt Medienkonvergenz der Johannes Gutenberg-Universität Mainz gefördert; im Jahr 2019 wurde die Datenerhebung aus 
Eigenmitteln der beteiligten Wissenschaftlerinnen und Wissenschaftler finanziert. Es handelt sich um Trenddaten, es wurden also jedes Jahr unterschiedliche Personen befragt. Nach dem ADM-Telefonstichprobensystem (vgl. Möhring und Schlütz 2010, S. 130-131) generierten wir eine Zufallsstichprobe von N=1200 Personen, auf Haushaltsebene kam die Last-Birthday-Methode zum Einsatz (vgl. Möhring und Schlütz 2010, S. 31). Die Interviews dauerten im Durchschnitt $25 \mathrm{~min}$, durchgeführt wurden sie von einem Meinungsforschungsinstitut. Soweit nicht anders vermerkt, haben wir die Antworten der Befragten auf fünfstufigen Likert-Skalen erhoben ( $1=$ niedrige Ausprägung bzw. hohe Ablehnung bis 5=hohe Ausprägung bzw. hohe Zustimmung).

Unter Medienvertrauen verstehen wir in der Langzeitstudie das globale Vertrauen in die Medien als gesellschaftliche Institution. Es wird nicht weiter unterschieden oder spezifiziert, welche Medien genau damit gemeint sind - die Befragten sollen analog zum Konzept von Tsfati (2003b, S. 160) die Fragen in Hinblick auf die Medien beantworten, mit denen sie in ihrem Alltag Kontakt haben, die sie kennen und deren Nutzung sie gewöhnt sind (vgl. Jackob 2012, S. 116). Auf diese Weise repräsentieren die Antworten der Befragten die in der Bevölkerung existierenden Vorstellungen. Entsprechend haben wir Medienvertrauen mit drei seit Langem etablierten Items gemessen (vgl. Jakobs et al. 2021; Schultz et al. 2020; Jackob et al. 2019b), die anschließend zu einem Index zusammengefasst wurden: „Man kann den Medien vertrauen“, „Man kann sich auf die Medien verlassen“ und „Wenn es um wirklich wichtige Dinge geht - etwa Umweltprobleme, Gesundheitsgefahren, politische Skandale und Krisen -, kann man den Medien vertrauen“ (Reliabilität Cronbach's Alpha 2017=0,74, 2018=0,77, 2019=0,83).

Die soziodemografischen Faktoren Alter, Bildung und Geschlecht haben wir mit entsprechenden Fragen erhoben.

Bei den Einstellungen zu Politik haben wir das politische Interesse mit der Frage „Wie interessiert sind Sie im Allgemeinen an der Politik?“ erfasst. Politikverdrossenheit haben wir mit der Zustimmung zu zwei Aussagen erfragt: „Die Politiker kümmern sich nicht darum, was Leute wie ich denken“ und „Politiker reden zu viel und handeln zu wenig“. Diese haben wir zu einem Index zusammengefasst (Reliabilität Spearman-Brown-Koeffizient 2017=0,55, $2018=0,64,2019=0,65$ ). Demokratiezufriedenheit haben wir mit der Zustimmung zur Aussage ,Ich bin mit der Art und Weise, wie die Demokratie in Deutschland funktioniert, zufrieden“ erhoben.

Die Mediennutzung haben wir ermittelt, indem die Befragten nach der Nutzungshäufigkeit verschiedener Mediengattungen und Internetangebote gefragt wurden (täglich oder fast täglich, ein- oder mehrmals pro Woche, ein- oder mehrmals im Monat, seltener als einmal im Monat, nie): Öffentlich-rechtliches Fernsehen, privates Fernsehen, Tageszeitungen, Boulevardzeitungen, Online-Angebote etablierter Medien, alternative Nachrichtenseiten, Nachrichten auf sozialen Netzwerken, Nachrichten auf Videoplattformen.

Den Verschwörungsglauben haben wir mit der Zustimmung zu drei Verschwörungserzählungen erhoben, in denen die Medien selbst nicht als primäre Akteure vorkommen. So ergibt sich ein medienunabhängiger Indikator für verschwörungsideologische Denkmuster. Eine klassische Verschwörungserzählung ist die Aussage: „Die Anschläge auf das World Trade Center am 11. September 2001 wurden 
von den USA selbst inszeniert.“ Neuere Verschwörungserzählungen beziehen sich auf aktuellere Entwicklungen: „Die unkontrollierte Flüchtlingszuwanderung ist eine neue Strategie zur Abschaffung des deutschen Volkes“ und „Flugzeuge versprühen im Auftrag von Regierungen Chemikalien, um Wetter und Klima zu verändern“. Die Zustimmung zu diesen Verschwörungserzählungen wurde zu einem MittelwertIndex zusammengefasst (Reliabilität Cronbach's Alpha 2017=0,52, 2018=0,67, $2019=0,67)$.

Interpersonales Vertrauen haben wir mit zwei Items gemessen, die anschließend zu einem Index zusammengefasst wurden: „Im Allgemeinen kann man den Menschen vertrauen“ und „Ich bin davon überzeugt, dass die meisten Menschen gute Absichten haben“ (Reliabilität Spearman-Brown-Koeffizient 2017=0,72, $2018=0,72$, $2019=0,69)$.

Zur Beantwortung der Forschungsfragen haben wir in den drei Datensätzen der Jahre 2017, 2018 und 2019 Regressionsanalysen gerechnet. Medienvertrauen stellte dabei die abhängige Variable dar, die gemessenen Einflussfaktoren bildeten die unabhängigen Variablen. Die Einflussfaktoren haben wir dabei blockweise eingespeist (Variableneinschluss-Methode „Einschluss“), sodass sowohl der Einfluss einer einzelnen Variablengruppe als auch der Einfluss aller Variablen gemeinsam aufgezeigt werden kann. Auf diese Weise sind pro Jahr vier Modelle entstanden. Die soziodemografischen Merkmale wurden als grundlegende Personenmerkmale zuerst in die blockweisen Regressionen eingegeben. Diese dienen als Kontrollvariablen und müssen zuerst in die Regressionsgleichungen aufgenommen werden, damit ihr Einfluss nicht von anderen Faktoren überdeckt wird. Als weiteres recht grundlegendes Merkmal der Rezipientinnen und Rezipienten folgte die Mediennutzung, dann kamen die komplexeren Einstellungs-Variablen hinzu - zunächst in Form der Einstellungen zu Politik, zum Schluss in Form von Verschwörungsglauben und interpersonalem Vertrauen.

Im Jahr 2018 haben wir zudem weitere Variablen erhoben, sodass der Datensatz aus diesem Jahr umfangreicher ist als der von 2017 und 2019. Dass die zusätzlichen Variablen nur im Jahr 2018 erhoben wurden, hatte forschungsökonomische Gründe. Um zu untersuchen, ob zusätzliche Variablen einen Erklärungsbeitrag zu Medienvertrauen leisten können, haben wir eine ergänzende Regressionsanalyse gerechnet, bei der ebenfalls Medienvertrauen die abhängige Variable darstellte. Neben den oben beschriebenen Einflussfaktoren wurden als unabhängige Variablen in diese Berechnung zusätzliche Konstrukte einbezogen, die in der Vertrauensforschung bisher keine Rolle spielten und daher nicht aus der Literatur hergeleitet werden konnten. Die Konstrukte haben wir auf der Suche nach möglichen Gründen für Veränderungen im Vertrauen und vor dem Hintergrund der Diskussion um Medienvertrauen im Jahr 2018 in den Fragebogen aufgenommen. Sie werden daher nun auf ihren Einfluss auf Medienvertrauen untersucht:

Die wirtschaftliche Situation haben wir erhoben, indem die Befragten angeben sollten, wie sehr sie mit ihrer aktuellen wirtschaftlichen Lage zufrieden sind. AuBerdem wurden sie gebeten, einzuschätzen, ob sich ihre wirtschaftliche Situation zukünftig eher verbessern oder eher verschlechtern wird.

Das Wissen über Medien haben wir abgefragt, indem die Befragten bei vier Aussagen über Medien und Journalismus angeben sollten, ob diese richtig oder falsch 
sind (z. B. „Der Staat prüft in Deutschland, ob jemand Journalist werden kann“ oder „Journalisten sind gesetzlich dazu verpflichtet, den Wahrheitsgehalt ihrer Beiträge zu prüfen“). Aus den Antworten wurde ein Index berechnet, der die Anzahl der richtig beantworteten Fragen angibt und der somit Werte zwischen 0 und 4 annehmen kann.

Medienentfremdung haben wir mit der Zustimmung zu vier Aussagen erhoben, z.B. „Die Medien haben den Kontakt zu Menschen wie mir verloren“ oder „Die Themen, die mir wichtig sind, werden von den Medien gar nicht ernst genommen“. Daraus wurde ein Mittelwert-Index gebildet (Reliabilität Cronbach's Alpha =0,78).

Zudem haben wir das Vertrauen in Institutionen abgefragt. Dazu wurden den Befragten sechs gesellschaftliche Institutionen genannt: Kirchen, Wissenschaft, Politik, Justiz, Unternehmen und Bundeswehr. Sie sollten jeweils angeben, wie sehr sie diesen vertrauen. Da es uns um die generelle Einschätzung der Vertrauenswürdigkeit gesellschaftlicher Institutionen geht, fassten wir diese zu einem Mittelwert-Index zusammen. Eine explorative Faktorenanalyse (Hauptkomponentenanalyse, Varimax-Rotation) bestätigte dieses Vorgehen und kam zu einer Ein-FaktorLösung (KMO=0,80; erklärte Gesamtvarianz: 42,06\%) (Reliabilität Cronbach's Alpha $=0,72)$.

Schließlich haben wir die Partizipationsbereitschaft der Befragten erhoben. Dazu wurden ihnen sechs Formen der Beteiligung genannt, und sie sollten angeben, ob sie so etwas künftig tun werden oder nicht (z. B. ,meine Meinung bei Diskussionen über Politik äußern“, „,bei den nächsten Wahlen meine Stimme abgeben“ oder „,ür meine Ideen auf die Straße gehen, auch gegen den Widerstand von Ordnungskräften“). Das Ausmaß der Partizipationsbereitschaft, unabhängig von der konkreten Beteiligungsform, wurde in Form eines Summen-Index in die Regression eingespeist, der Werte zwischen 0 (keine der Optionen bejaht) und 6 (alle Optionen bejaht) annehmen kann (Reliabilität Kuder-Richardson-20-Koeffizient=0,59).

In allen Regressionsanalysen waren die Voraussetzungen zur Berechnung multipler Regressionen erfüllt - insbesondere lag keine Multikollinearität vor, Homoskedastizität und Normalverteilung der Residuen waren stets gegeben.

\section{Ergebnisse}

Tab. 1 zeigt die Regressionsmodelle für die Jahre 2017, 2018 und 2019. Für jedes Jahr gibt es vier Blöcke, in denen die Variablen nach und nach eingespeist wurden (Modelle 2017/1, 2017/2, usw.).

Im ersten Block haben wir die soziodemografischen Faktoren Alter, Bildung und Geschlecht in das Modell einbezogen. Dieses Modell ist in allen drei Jahren signifikant und erklärt 1-2\% der Varianz im Medienvertrauen. Das Alter beeinflusst in diesem Modell Medienvertrauen nicht signifikant. Die Bildung zeigt einen hochsignifikanten Einfluss: Jeweils weisen formal höher gebildete Befragte ein größeres Medienvertrauen auf als formal niedrig Gebildete. Das Geschlecht zeigt in zwei der drei Jahre einen leicht negativen Einfluss - Frauen gaben hier ein niedrigeres Medienvertrauen an als Männer. Die Beta-Werte zeigen, dass der Einfluss der Bildung auf das Medienvertrauen größer ist als der des Geschlechts. 


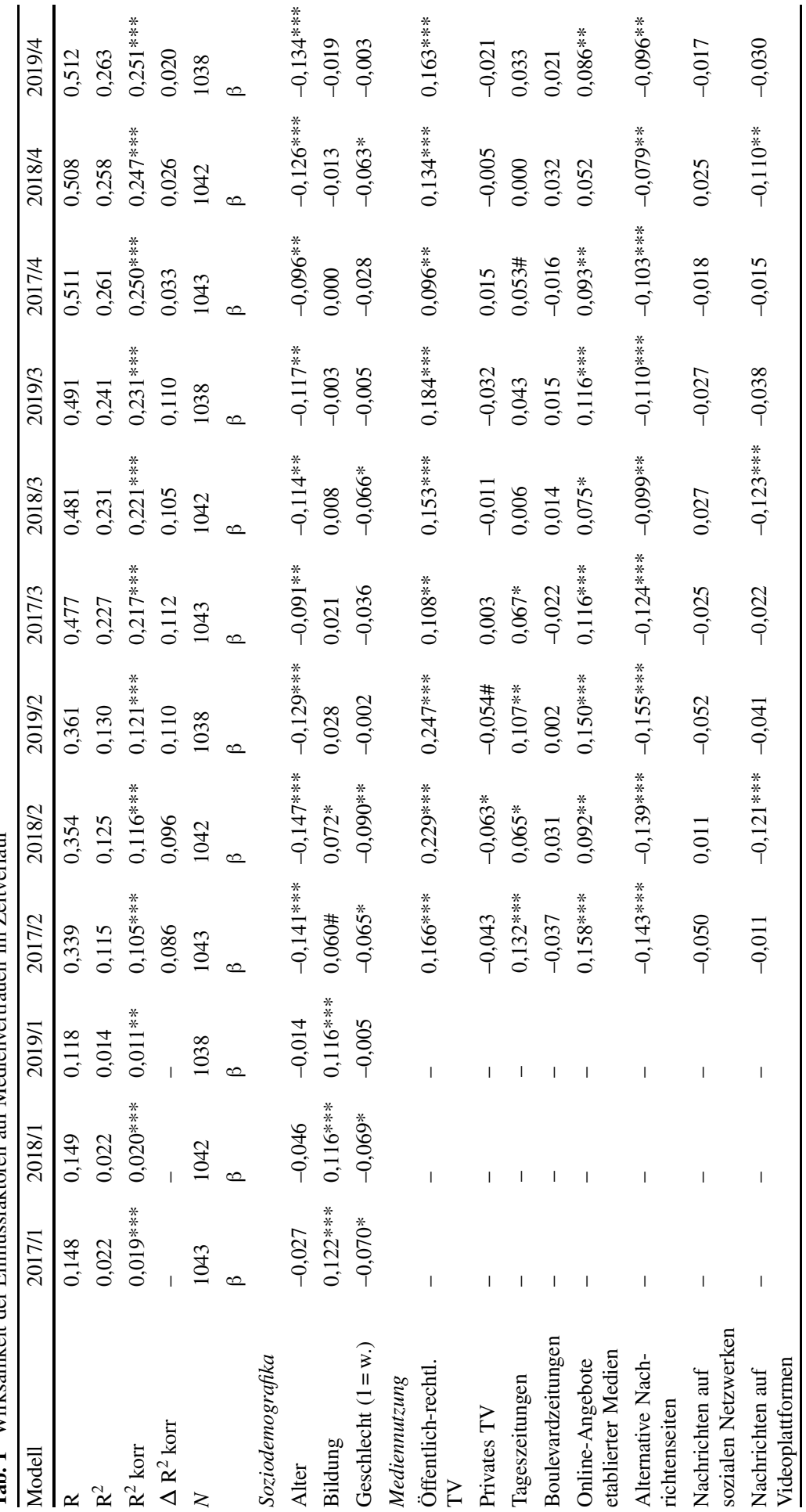




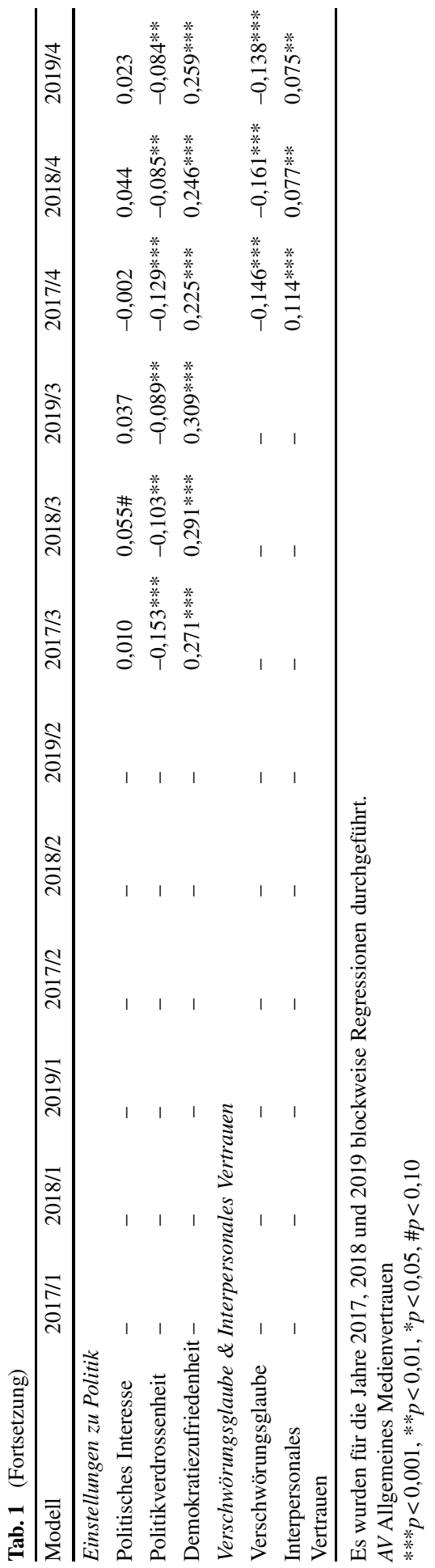


Im zweiten Block haben wir die Mediennutzung hinzugenommen. Auch hier sind die Modelle für alle drei Jahre signifikant und erklären etwa 11-12\% der Varianz im Medienvertrauen - die Mediennutzung steigert die Erklärungskraft der Modelle somit deutlich. In allen drei Jahren zeigt sich nun ein signifikanter und starker Effekt des Alters: Ältere Befragte geben ein niedrigeres Medienvertrauen an als Jüngere. Der Einfluss der Bildung schwächt sich etwas ab und verschwindet in einem Jahr sogar ganz. Der Einfluss des Geschlechts bleibt unverändert. Bei der Mediennutzung zeigt sich, dass vor allem die Nutzung von öffentlich-rechtlichem Fernsehen signifikant und stark mit Medienvertrauen zusammenhängt: Wer diese Angebote häufiger nutzt, hat ein größeres Medienvertrauen. Auch für Tageszeitungen zeigt sich ein solcher Effekt, wenngleich etwas schwächer als bei den Öffentlich-Rechtlichen. Weiterhin geht auch eine häufige Nutzung der Online-Angebote etablierter Medien mit einem größeren Medienvertrauen einher. Der Einfluss dieser drei Mediengattungen zeigt sich über alle drei Jahre hinweg. Die Beta-Werte weisen für die Nutzung des öffentlich-rechtlichen Rundfunks den stärksten Einfluss auf. Der Einfluss der Nutzung von Tageszeitungen oder der Online-Angebote etablierter Medien ist schwächer.

Ein geringeres Medienvertrauen weisen in zwei Befragungsjahren diejenigen auf, die häufiger privates Fernsehen nutzen - die Beta-Werte deuten hier jedoch auf einen schwachen Einfluss hin. In einem Jahr hängt auch die Nutzung von Nachrichten auf Videoplattformen mit einem geringeren Medienvertrauen zusammen. In allen drei Jahren zeigt sich ein Einfluss der Nutzung von alternativen Nachrichtenseiten, die mit einem geringeren Medienvertrauen einhergeht. Die Stärke dieses Effekts ist im Mittel in etwa vergleichbar mit dem der Nutzung von Tageszeitungen und Online-Angeboten traditioneller Medien. Die Nutzung von Boulevardzeitungen und Nachrichten auf sozialen Netzwerken steht in keinem Zusammenhang mit Medienvertrauen.

Im dritten Block haben wir die Einstellungen zu Politik als Erklärungsvariablen hinzugefügt. Die Modelle für alle drei Jahre sind signifikant und erklären etwa 22-23\% der Varianz im Medienvertrauen - die Einstellungen zu Politik steigern die Erklärungskraft also nochmals deutlich. Bei den soziodemografischen Variablen bleibt der Einfluss des Alters bestehen, schwächt sich jedoch leicht ab. Die Bildung zeigt keinen signifikanten Einfluss mehr, und das Geschlecht beeinflusst nur noch in einem Erhebungsjahr das Medienvertrauen. Bei der Mediennutzung schwächt sich der Einfluss der Nutzung öffentlich-rechtlichen Fernsehens etwas ab, genauso wie der Einfluss der Online-Angebote etablierter Medien. Tageszeitungen zeigen nur noch in einem Jahr einen signifikanten Effekt, und auch der Einfluss des privaten Fernsehens verschwindet. Etwas abgeschwächt ist nun auch der Einfluss der Nutzung alternativer Nachrichtenseiten; die Nutzung von Nachrichten auf Videoplattformen ist weiterhin in einem Jahr signifikant. Bei den Einstellungen zu Politik weist das politische Interesse nur in einem Erhebungsjahr einen tendenziell signifikanten Einfluss auf, der Beta-Wert deutet auf einen eher schwachen Einfluss hin: Befragte mit einem größeren politischen Interesse haben hier ein größeres Medienvertrauen. Signifikant und sehr stark ist in allen Jahren der Zusammenhang zwischen Medienvertrauen und Demokratiezufriedenheit - wer mit der Demokratie in der Bundesrepublik zufriedener ist, weist ein höheres Medienvertrauen auf. Die Beta-Werte weisen die 
Demokratiezufriedenheit als stärksten Einflussfaktor in diesem Modell aus. Das Gegenteil trifft auf diejenigen mit einer größeren Politikverdrossenheit $\mathrm{zu}$ - diese steht in allen drei Jahren in einem signifikanten Zusammenhang mit Medienvertrauen, der etwas schwächer ist als der der Demokratiezufriedenheit.

Im vierten Block haben wir schließlich noch den Verschwörungsglauben und das interpersonale Vertrauen im Modell ergänzt. Die Regressionen sind in allen drei Jahren signifikant und erklären nun etwa $25 \%$ der Varianz im Medienvertrauen der letzte Variablenblock hat also nochmals zu einer Steigerung der Varianzaufklärung beigetragen. Bei den soziodemografischen Faktoren ergeben sich gegenüber dem Vormodell keine Änderungen. Bei der Mediennutzung schwächen sich manche Zusammenhänge etwas ab, die grundsätzlichen Muster bleiben aber bestehen. Bei den Einstellungen zu Politik gilt das gleiche. Der Verschwörungsglaube zeigt einen signifikanten und starken Zusammenhang mit Medienvertrauen - wer Verschwörungserzählungen eher Glauben schenkt, hat ein niedrigeres Medienvertrauen. Umgekehrt führt ein größeres interpersonales Vertrauen zu einem höheren Medienvertrauen - dieser Effekt ist nicht ganz so stark wie der des Verschwörungsglaubens. Hinsichtlich der Beta-Werte bleibt in diesen Modellen die Demokratiezufriedenheit der stärkste Einflussfaktor, gefolgt vom Verschwörungsglauben. ${ }^{2}$

Forschungsfrage 1 bezog sich auf die sozidemografischen Faktoren Alter, Geschlecht und Bildung. Unsere Analyse hat gezeigt, dass diese Medienvertrauen beeinflussen - wenn auch nicht in besonders großem Maße. Dieser Einfluss wird in komplexeren Modellen allerdings z.T. von anderen Variablen überlagert.

Forschungsfrage 2 zielte auf die Nutzung unterschiedlicher Mediengattungen ab. Hier wird erkennbar, dass die Mediennutzung das Medienvertrauen beeinflusst. Dabei wirkt sich die Nutzung verschiedener Mediengattungen unterschiedlich auf das Medienvertrauen aus. Nur zwei der abgefragten Mediengattungen beeinflussten das Medienvertrauen gar nicht, nämlich Boulevardzeitungen und Nachrichten aus sozialen Netzwerken. Die Einflüsse zeigten sich bei den anderen Mediengattungen zumeist konstant über die Jahre hinweg - lediglich die Nutzung von Nachrichten auf Videoplattformen zeigte nur in einem Erhebungsjahr einen Einfluss. Der Einfluss der Mediennutzung wurde in komplexeren Modellen z. T. ebenfalls von anderen Variablen überlagert.

\footnotetext{
2 In den komplexesten Regressionsmodellen aus den Jahren 2017, 2018 und 2019 (jeweils Modell 4) haben wir zusätzlich bei ausgewählten Einflussfaktoren einen paarweisen Test auf Gleichheit der Regressionskoeffizienten vorgenommen. Hierfür haben wir solche Einflussfaktoren ausgewählt, die statistisch signifikant sind und bei denen sich in den drei Jahren deutliche Veränderungen in Signifikanz und/oder Effektstärke zeigten. Dies traf auf die Nutzung des öffentlich-rechtlichen Fernsehens, der Online-Angebote etablierter Medien sowie von Videoplattformen zu. Den Vergleich haben wir gemäß den Ausführungen von Paternoster et al. (1998) vorgenommen. Bei der Nutzung des öffentlich-rechtlichen Fernsehens unterscheiden sich die Regressionskoeffizienten trotz deutlicher Schwankungen im Beta-Wert nicht voneinander. Bei der Nutzung der Online-Angebote etablierter Medien unterscheiden sich die Regressionskoeffizienten von 2017 und 2019 sowie von 2018 und 2019 voneinander. Die Nutzung der Online-Angebote zeigt 2017 und 2019 einen signifikanten Effekt mit ähnlicher Effektstärke, der für beide Jahre stärker ausfällt als 2018. Bei der Nutzung von Videoplattformen unterscheiden sich die Regressionskoeffizienten der Jahre 2017 und 2018 voneinander. Die Nutzung von Videoplattformen zeigt 2018 einen stärkeren Einfluss als in den anderen beiden Jahren. Erklärungen für die unterschiedlichen Größen der Regressionskoeffizienten können wir anhand unserer Daten nicht liefern.
} 
Forschungsfrage 3 behandelte die Einstellungen zu Politik. Diese beeinflussen das Medienvertrauen: Demokratiezufriedenheit und Politikverdrossenheit beeinflussten das Medienvertrauen durchgängig und stark, der Einfluss des politischen Interesses wurde im komplexeren Modell ebenfalls von anderen Variablen überlagert.

Forschungsfrage 4 bezog sich auf den Verschwörungsglauben, der mit einem niedrigeren Medienvertrauen einhergeht. Dieser Einfluss war stark und über die Zeit konstant. Gleiches gilt für das in Forschungsfrage 5 angesprochene Vertrauen - je höher es ist, desto größer ist das Medienvertrauen. Auch dieser Einfluss war über die Zeit konstant.

In einem weiteren Regressionsmodell haben wir im Datensatz des Jahres 2018 zusätzlich zu den über die Zeit analysierten Einflussfaktoren weitere Variablen berücksichtigt, die nur in diesem Erhebungsjahr erfasst wurden. Für das Jahr 2018 lag somit ein umfangreicherer Datensatz als für die Jahre 2017 und 2019 vor. Ziel der zusätzlichen Analyse ist es, aufzuzeigen, ob es über die bisher betrachteten, aus der Theorie hergeleiteten Einflussfaktoren hinaus weitere Konstrukte gibt, die in einem Zusammenhang mit Medienvertrauen stehen und daher von der künftigen Forschung berücksichtigt werden sollten. Die im Jahresvergleich betrachteten Regressionsmodelle wiesen eine Varianzaufklärung von maximal rund $25 \%$ auf - Ziel dieser zusätzlichen Analyse ist es nun, herauszufinden, ob weitere Variablen einen deutlichen Erklärungsbeitrag zu Medienvertrauen liefern können. Die Vorgehensweise ist hier also empiriegeleitet - den Regressionsmodellen aus den Jahren 2017-2019 wird ein erweitertes Modell aus dem Jahr 2018 gegenübergestellt, um zu prüfen, ob weitere Variablen zur Maximierung der Varianzaufklärung beitragen können. Tab. 2 zeigt dieses Regressionsmodell (Modell ,2018 alle Var.“).

Das Modell ist signifikant und erklärt rund $40 \%$ der Varianz im Medienvertrauen - mithilfe der zusätzlich betrachteten Einflussfaktoren konnte die Erklärungskraft also nochmal deutlich gesteigert werden. Rund $15 \%$ Varianzaufklärung kamen hin$\mathrm{zu}$ - die zusätzlich berücksichtigten Variablen tragen so dazu bei, Medienvertrauen noch besser vorherzusagen und somit auch verstehen zu können. Vergleichsmaßstab für dieses erweiterte Modell ist das Modell 4 von 2018 aus Tab. 1, das wir zum Vergleich in Tab. 2 dem erweiterten Modell aus dem Jahr 2018 gegenüberstellen.

Im Modell mit allen Variablen ergeben sich bei den Soziodemografika keine Veränderungen. Bei der Mediennutzung zeigt sich ein zusätzlicher Einfluss der Nutzung von Boulevardzeitungen - je häufiger deren Nutzung, desto größer ist das Medienvertrauen. Der Einfluss der Nutzung öffentlich-rechtlichen Fernsehens schwächt sich etwas ab, ebenso der der Nutzung von Videoplattformen. Bei den Einstellungen zu Politik schwächt sich der Einfluss der Demokratiezufriedenheit deutlich ab. Der Einfluss der Politikverdrossenheit verschwindet. Der Einfluss des Verschwörungsglaubens ist etwas schwächer, derjenige des interpersonalen Vertrauens leicht stärker.

Von den neu hinzugekommenen Variablen zeigen die Zufriedenheit mit der wirtschaftlichen Situation und die Prognose der künftigen Entwicklung der eigenen wirtschaftlichen Situation einen signifikanten Effekt - je größer die Unzufriedenheit ist und je negativer die künftigen Entwicklungen gesehen werden, desto geringer ist das Medienvertrauen. Die Beta-Werte weisen hier auf einen eher schwachen Effekt hin. Das Wissen über Medien steht nicht in einem Zusammenhang mit Medienver- 
Tab. 2 Regressionsmodell Medienvertrauen mit zusätzlichen Variablen (Jahr 2018)

\begin{tabular}{lll}
\hline Modell & $\begin{array}{l}2018 / 4 \text { (Modell aus } \\
\text { Tab. 1 zum Vgl.) }\end{array}$ & $\begin{array}{l}2018 \text { alle Var. (neues Modell mit } \\
\text { zusätzlichen Variablen) }\end{array}$ \\
\hline $\mathrm{R}^{2}$ korr & $0,247 * * *$ & $0,405 * * *$ \\
$N$ & 1042 & 807 \\
& $\beta$ & $\beta$
\end{tabular}

\section{Soziodemografie}

Alter

$\begin{array}{ll}-0,126 * * * & -0,135 * * * \\ -0,013 & -0,041 \\ -0,063 * & -0,069 *\end{array}$

Geschlecht (1= weibl.)

$0,134 * * *$

$0,077 *$

Öffentlich-rechtliches TV

$-0,005$

0,007

Privates TV

0,000

$-0,005$

Tageszeitungen

0,032

$0,090 * *$

Online-Angebote etablierter Medien

0,052

$-0,005$

Alternative Nachrichtenseiten

$-0,060^{*}$

Nachrichten auf sozialen Netzwer-

0,025

0,005

ken

Nachrichten auf Videoplattformen

$-0,110 * *$

$-0,074 *$

Einstellungen zu Politik

Politisches Interesse

0,044

$-0,015$

Politikverdrossenheit

$-0,085 * *$

0,053

Demokratiezufriedenheit

$0,246 * * *$

$0,084 *$

Verschwörungsglaube \& Interpersonales Vertrauen
Verschwörungsglaube
$-0,161 * * *$
$-0,123 * *$
Interpersonales Vertrauen
$0,077 * *$
$0,107 * * *$

Wirtschaftliche Situation

Zufriedenheit mit wirtsch. Situation

$-0,072 *$

Künftige Entwicklung wirtsch.

$-0,063^{*}$

Situation

Einstellungen zu und Wissen über Medien

Medienwissen (Summen-Index 0-4) -

0,035

Medienentfremdung

Institutionen-Vertrauen

Vertrauen in gesellschaftliche Insti-

$0,307 * * *$ tutionen

Partizipationsbereitschaft

Partizipationsbereitschaft (Summen-

$0,107 * *$

Index 0-6)

$A V$ Allgemeines Medienvertrauen

*** $p<0,001,{ }^{* *} p<0,01,{ }^{*} p<0,05, \# p<0,10$ 
trauen. Dieser ist bei der Medienentfremdung hingegen hochsignifikant und stark: Wer sich von den Medien entfremdet fühlt, hat ein niedrigeres Medienvertrauen.

Das Vertrauen in Institutionen hängt mit Medienvertrauen zusammen: Menschen, die anderen gesellschaftlichen Institutionen vertrauen, weisen ein höheres Medienvertrauen auf. Dieser Beta-Wert ist der höchste aller durchgeführten Regressionsmodelle. Die Partizipationsbereitschaft steht ebenfalls in einem Zusammenhang mit Medienvertrauen: Wer angibt, sich künftig vermehrt politisch engagieren zu wollen, weist ein höheres Medienvertrauen auf. Dieser Effekt hat eine mittlere Stärke.

Es hat sich also herausgestellt, dass auch die eigene wirtschaftliche Situation, die Entfremdung von den Medien, das Vertrauen in andere gesellschaftliche Institutionen und die Partizipationsbereitschaft zusätzliche Erklärungsbeitrage zu Medienvertrauen leisten und die Erklärungskraft von Modellen nochmals deutlich steigern können.

\section{Diskussion und Fazit}

In drei Querschnitts-Befragungen in den Jahren 2017 bis 2019 haben wir untersucht, welche Eigenschaften der Rezipientinnen und Rezipienten über einen längeren Zeitraum auf das Medienvertrauen der Deutschen einwirken und welche nicht. Dazu haben wir mit den Daten der drei Befragungswellen blockweise Regressionsanalysen durchgeführt. Dabei konnte jeweils maximal ein Viertel der Varianz im Medienvertrauen durch die untersuchten Einflussfaktoren erklärt werden, die Varianzaufklärung steigerte sich mit jedem hinzugefügten Variablenblock. Es zeigte sich, dass eine höhere formale Bildung, die Nutzung von öffentlich-rechtlichem Fernsehen, Tageszeitungen, den Online-Angeboten etablierter Medien sowie Zufriedenheit mit der Demokratie in der Bundesrepublik und interpersonales Vertrauen mit einem höheren Medienvertrauen zusammenhängen. In der zusätzlichen Regressionsanalyse mit den Daten aus dem Jahr 2018 zeigten Befragte mit einem größeren Vertrauen in gesellschaftliche Institutionen wie Politik, Wissenschaft oder Justiz ein höheres Medienvertrauen - ebenso diejenigen, die bereit sind, sich künftig verstärkt politisch zu engagieren. Bürgerinnen und Bürger, die vom Funktionieren der Demokratie überzeugt sind und zudem von der Vertrauenswürdigkeit ihrer Mitmenschen und der gesellschaftlichen Institutionen ausgehen, die sich zugleich aus dem öffentlich-rechtlichen Rundfunk, Tageszeitungen bzw. in etablierten Medien über das tagesaktuelle Geschehen informieren und höher gebildet sind, zeichnen sich durch ein größeres Maß an Medienvertrauen aus. Diese Bürgerinnen und Bürger sind also grundsätzlich sowohl dem Staat und seinen Institutionen als auch den Medien wohlgesonnen und bereit, sich am demokratischen Prozess zu beteiligen.

Ein höheres Alter, das weibliche Geschlecht, die Nutzung privaten Fernsehens und alternativer Nachrichtenseiten sowie teilweise von Nachrichten auf Videoplattformen hängen hingegen mit einem niedrigeren Medienvertrauen zusammen, ebenso wie Politikverdrossenheit und der Verschwörungsglaube. In der zusätzlichen Regressionsanalyse mit allen im Jahr 2018 erhobenen Variablen zeigt sich weiterhin, dass Menschen mit einer größeren Entfremdung von den Medien sowie mit einer pessimistischen Einschätzung der eigenen aktuellen und künftigen wirtschaftlichen 
Situation ebenfalls ein geringeres Medienvertrauen haben. Diejenigen, die mit der Politik und den Medien unzufrieden sind und sich von ihnen nicht angemessen repräsentiert fühlen, die zugleich an Verschwörungen sinistrer Mächte glauben und ihre Informationen zudem vermehrt auf alternativen Nachrichtenseiten abseits der Mainstream-Medien beziehen, zeichnen sich also durch ein geringeres Maß an Medienvertrauen aus.

Aus demokratietheoretischer Hinsicht ist es wichtig zu wissen, wie groß die oben beschriebene Gruppe der Bürger ist, die von Medien und Politik entfremdet sind und diesen misstrauisch gegenüberstehen. Andere Analysen unserer Daten lassen den Schluss zu, dass sich dieser harte Kern in den vergangenen Jahren verfestigt hat und bei rund $20 \%$ der Bürgerinnen und Bürger liegt (vgl. Jackob et al. 2019b; Schultz et al. 2020). Dies stellt einen nicht unerheblichen Anteil der Bevölkerung dar. Diese Personengruppe kann also die Zielgruppe derjenigen sein, die sich bemühen möchten, das Vertrauen in Medien zu steigern. Gleichzeitig erwecken diese Bürgerinnen und Bürger jedoch den Eindruck, von der Politik und den etablierten Medien nur schwer erreichbar zu sein - hierin liegt also zum einen die große gesellschaftliche Herausforderung, diese Personengruppe nicht zu verlieren, und gleichzeitig ein Gefährdungspotenzial für die Demokratie, wenn solche Bürgerinnen und Bürger sich in Gruppierungen mit Gleichgesinnten zurückziehen. In unserer Datenerhebung im November und Dezember 2020 war coronabedingt der Anteil an Menschen mit medienzynischen Denkmustern deutlich zurückgegangen (vgl. Jakobs et al. 2021). Ob diese Entwicklung von Dauer sein wird oder ob diese Einstellung nach der CoronaKrise wieder auf das Vorkrisen-Niveau ansteigt, ist derzeit noch nicht absehbar.

Im Hinblick auf die Stabilität des Einflusses von Merkmalen der Rezipientinnen und Rezipienten auf das Medienvertrauen bieten die Ergebnisse unserer Untersuchung genuine Einblicke in Bereiche, zu denen die vorherige, größtenteils auf einmalig durchgeführten Befragungen beruhende Forschung teils widersprüchliche Erkenntnisse erlangt hat. Die blockweise durchgeführten Regressionsanalysen trugen dabei zur Aufklärung bei - denn sie verdeutlichten, dass in den weniger komplexen Modellen Variablen einen Einfluss zeigten, der in komplexeren Modellen nicht mehr auftauchte und deren Einfluss somit vermutlich von den hinzugefügten Variablen überdeckt wurde. Dies erklärt, warum z. B. zum Einfluss der Soziodemografika auf Medienvertrauen ein so heterogener Forschungsstand vorliegt: Ob diese Variablen einen Einfluss zeigen oder nicht, hängt schlicht und ergreifend davon ab, welche einflussreicheren Variablen in den vorliegenden Studien noch berücksichtigt wurden. Zumeist handelt es sich bei diesen besonders einflussreichen Variablen um politisch-ideologische Einstellungsmuster. Auf diese Weise trägt unsere Studie dazu bei, das Forschungsfeld der Beeinflussung von Medienvertrauen zu bereichern. Da wir unsere Analysen nicht nur zu einem Messzeitpunkt durchgeführt haben, sondern in mehreren Jahren, konnten wir außerdem Aussagen darüber treffen, welche Personenmerkmale nur einmalig einen Einfluss auf Medienvertrauen zeigten und bei welchen Merkmalen sich dieser Einfluss stabil über die Zeit nachweisen lässt. Dieses Alleinstellungsmerkmal unserer Untersuchung ermöglicht der kommunikationswissenschaftlichen Vertrauensforschung künftig klarere Erkenntnisse über die Merkmale, die Medienvertrauen beeinflussen, als sie in der deutschen Forschungslandschaft bisher vorlagen. 
In unseren Analysen war überraschend, dass das Alter als Einflussfaktor auf Medienvertrauen erst dann einen Einfluss zeigt, wenn die Mediennutzung zur Analyse hinzugefügt wird. Möglicherweise hängt das mit den unterschiedlichen Mediennutzungsmustern älterer und jüngerer Befragter zusammen (vgl. Breunig et al. 2020). Die Nutzung von Boulevardzeitungen steht nur im zusätzlichen Regressionsmodell aus dem Jahr 2018 im Zusammenhang mit Medienvertrauen und ansonsten nicht - die Nutzung von Nachrichten auf Videoportalen zeigt nur in allen Regressionen aus dem Jahr 2018 einen Einfluss und ansonsten nicht. Bezüglich dieser Mediengattungen besteht also weiterer Forschungsbedarf. Im dritten Block, in dem wir die Einstellungen zu Politik hinzugefügt haben, verschwindet der Einfluss der Bildung. Diese scheint mit diesen Merkmalen also zusammenzuhängen, und ihr Einfluss im Regressionsmodell wird von dem der Einstellungen zu Politik überlagert. Die Nutzung von Tageszeitungen und des privaten Fernsehens verlieren im gleichen Block ihren Einfluss - dies hängt also vermutlich ebenfalls mit den Einstellungen zu Politik zusammen.

Die zusätzliche Regressionsanalyse mit weiteren Variablen aus dem Jahr 2018 hat aufgezeigt, dass es über die bisher in der Forschung standardmäßig analysierten Einflussfaktoren hinaus noch weitere Konstrukte gibt, die einen großen Einfluss auf Medienvertrauen haben können. Die eigene wirtschaftliche Situation spielt dabei ebenso eine wichtige Rolle wie die wahrgenommene Entfremdung von den Medien sowie das Vertrauen in gesellschaftliche Institutionen und die Partizipationsbereitschaft. Durch das Hinzuziehen dieser Erklärungsvariablen stieg die Varianzaufklärung noch einmal deutlich an. Für die Medienvertrauensforschung erscheint es daher lohnend, künftig noch weitere Variablen auf ihren Einfluss auf Medienvertrauen hin zu untersuchen. In der zusätzlichen Regressionsanalyse wies die Politikverdrossenheit keinen Einfluss auf Medienvertrauen mehr auf - auch der Einfluss dieser Variable scheint also von anderen Variablen überlagert zu werden und hängt möglicherweise mit der Wahrnehmung der eigenen wirtschaftlichen Situation, der Partizipationsbereitschaft sowie dem Vertrauen in andere Institutionen zusammen.

Zwar konnten wir die hier untersuchten Einflussfaktoren auf Medienvertrauen lediglich im Sinne von Trendanalysen und nicht anhand einer Panelstudie untersuchen - wir konnten dabei jedoch verdeutlichen, welche Einflussfaktoren unabhängig von Messzeitpunkt, aktueller Stimmungslage oder Themenprävalenz einen durchgängigen, über die Jahre stabilen Einfluss auf das Medienvertrauen zeigten und bei welchen Faktoren dieser Einfluss eher instabil war, also vermutlich von Messzeitpunkt, der Stimmung zum Zeitpunkt der Befragung oder von Themen, über die in den Medien gerade berichtet wird, abhängen kann. Diese Erkenntnis unterstreicht die Notwendigkeit von Langzeitstudien, denn nur mit diesen sind solche Erkenntnisse möglich. Mithilfe von Panelstudien könnten solche Erkenntnisse künftig auf eine noch breitere Basis gestellt werden. In der Vertrauensforschung gibt es viele weitere Bereiche, in denen in den letzten Jahren vielfältige empirische Erkenntnisse gewonnen wurden, die mithilfe von Langzeitstudien auf ein empirisch gefestigteres Fundament gestellt werden könnten. Unter anderem würde sich für die Erforschung anderer Arten von Einflussfaktoren, wie Mediensystem-Variablen, gesellschaftliche Merkmale, wirtschaftliche Leistung oder politische Gegebenheiten, eine international vergleichende Perspektive anbieten. 
Unsere Messung von Medienvertrauen hat zudem das globale Vertrauen in die Medien betrachtet - hier könnten künftige Studien versuchen, mit differenzierteren und spezifischeren Messungen herauszufinden, ob andere Formen von Medienvertrauen durch andere Arten von Faktoren beeinflusst werden. Außerdem wäre es wichtig, einzelne der unabhängigen Variablen mit einer größeren Zahl von Indikatoren zu messen: Bei den allermeisten Variablen haben wir eine zufriedenstellende Reliabilität erreicht, in Einzelfällen kann diese noch gesteigert werden. Außerdem haben wir die betrachteten Einstellungen anhand bestimmter Konstrukte untersucht bei den politischen Einstellungen gibt es sicher neben politischem Interesse, der Demokratiezufriedenheit und der Politikverdrossenheit weitere Einstellungen, die Medienvertrauen beeinflussen können und die in künftigen Studien Berücksichtigung finden sollten. Dies könnte dazu beitragen, Erkenntnisse über Einflussfaktoren noch differenzierter und gründlicher zu untersuchen, als wir das getan haben. Ein möglicher weiterer Ansatz für die Medienvertrauensforschung könnte darin liegen, anstelle der klassischen soziodemografischen Variablen oder der hier untersuchten Einstellungsmerkmale den Ursprung von Vertrauensurteilen in der alltäglichen Lebenswelt der Menschen zu suchen und somit den lebensweltlichen Kontext der Menschen zu berücksichtigen, wie es Haschke (2016) für politisches Vertrauen mit einem milieutheoretischen Ansatz bereits umgesetzt hat. Eine weitere Limitation unserer Befunde ist, dass wir manche Merkmale nur in einem Erhebungsjahr untersucht haben. Ein wichtiges Merkmal unserer Langzeitstudie zu Medienvertrauen ist es, einerseits wichtige Kennzahlen zum Medienvertrauen konstant in jedem Jahr zu erheben. Andererseits ergänzen wir in jedem Jahr je nach aktueller gesellschaftlicher und wissenschaftlicher Diskussion Fragen, die im spezifischen Erhebungsjahr von Interesse sind - was aber dazu führt, dass Konstrukte aus Vorjahren gestrichen werden müssen, um die Befragten nicht mit einer zu langen Befragungsdauer zu überfordern. Denn die Befragungsdauer bei einer telefonischen Umfrage ist sehr limitiert. Dies führt bei Untersuchungen im Zeitverlauf zwangsläufig zu Einschränkungen, was die konstante Verfügbarkeit von Konstrukten über die Zeit betrifft.

Funding Open Access funding enabled and organized by Projekt DEAL.

Open Access Dieser Artikel wird unter der Creative Commons Namensnennung 4.0 International Lizenz veröffentlicht, welche die Nutzung, Vervielfältigung, Bearbeitung, Verbreitung und Wiedergabe in jeglichem Medium und Format erlaubt, sofern Sie den/die ursprünglichen Autor(en) und die Quelle ordnungsgemäß nennen, einen Link zur Creative Commons Lizenz beifügen und angeben, ob Änderungen vorgenommen wurden.

Die in diesem Artikel enthaltenen Bilder und sonstiges Drittmaterial unterliegen ebenfalls der genannten Creative Commons Lizenz, sofern sich aus der Abbildungslegende nichts anderes ergibt. Sofern das betreffende Material nicht unter der genannten Creative Commons Lizenz steht und die betreffende Handlung nicht nach gesetzlichen Vorschriften erlaubt ist, ist für die oben aufgeführten Weiterverwendungen des Materials die Einwilligung des jeweiligen Rechteinhabers einzuholen.

Weitere Details zur Lizenz entnehmen Sie bitte der Lizenzinformation auf http://creativecommons.org/ licenses/by/4.0/deed.de. 


\section{Literatur}

Bentele, G. (Hrsg.). (2008). Objektivität und Glaubwürdigkeit: Medienrealität rekonstruiert. Wiesbaden: VS.

Blöbaum, B. (2016). Key factors in the process of trust. On the analysis of trust under digital conditions. In B. Blöbaum (Hrsg.), Trust and communication in a digitized world. Models and concepts of trust research (S. 3-25). Cham: Springer.

Blöbaum, B. (2018). Bezugspunkte von Medienvertrauen. Ergebnisse einer explorativen Studie. Media Perspektiven, o.J.(12), 601-607.

Breunig, C., Handel, M., \& Kessler, B. (2020). Massenkommunikation 1964-2020: Mediennutzung im Langzeitvergleich. Ergebnisse der ARD/ZDF-Langzeitstudie. Media Perspektiven, o.J.(7-8), $410-432$.

Cuvalo, A. (2013). Institutional trust in the Croatian post-socialist context. CM Communication Management Quarterly, 26, 145-163.

Daniller, A., Allen, D., Tallevi, A., \& Mutz, D.C. (2017). Measuring trust in the press in a changing media environment. Communication Methods and Measures, 11, 76-85.

Dautrich, K., \& Hartley, T.H. (1999). How the news media fail American voters: causes, consequences and remedies. New York: Columbia University Press.

Denner, N., \& Peter, C. (2017). Der Begriff Lügenpresse in deutschen Tageszeitungen. Eine FramingAnalyse. Publizistik, 62, 273-297.

Donsbach, W., Rentsch, M., \& Schielicke, A.-M. (2009). Entzauberung eines Berufs. Was die Deutschen vom Journalismus erwarten und wie sie enttäuscht werden. Konstanz: UVK.

Engelke, K. M., Hase, V., \& Wintterlin, F. (2019). On measuring trust and distrust in journalism: reflection of the status quo and suggestions for the road ahead. Journal of Trust Research. https://doi.org/10. $1080 / 21515581.2019 .1588741$.

Fawzi, N. (2019). Untrustworthy news and the media as 'enemy of the people'? How a populist Worldview shapes recipients' attitudes toward the media. The International Journal of Press/Politics, 24, $146-164$.

Gambetta, D. (1988). Can we trust trust? In D. Gambetta (Hrsg.), Trust: making and braking cooperative relations (S. 213-237). Oxford: Basil Blackwell.

Granow, V., Jackob, N., Ziegele, M., Quiring, O., Schemer, C., \& Schultz, T. (2020). Interpersonales Vertrauen als Prädiktor für Medienvertrauen - Befunde der Mainzer Langzeitstudie. In A. Blome, T. Eberwein \& S. Averbeck-Lietz (Hrsg.), Medienvertrauen. Historische und aktuelle Perspektiven (S. 95-116). Berlin: De Gruyter.

Hagen, L. (2015). Nachrichtenjournalismus in der Vertrauenskrise. „Lügenpresse“ wissenschaftlich betrachtet: Journalismus zwischen Ressourcenkrise und entfesseltem Publikum. Communicatio Socialis, 48, 152-163.

Hanitzsch, T., van Dalen, A., \& Steindl, N. (2018). Caught in the nexus: a comparative and longitudinal analysis of public trust in the press. The International Journal of Press/Politics, 23, 3-23.

Haschke, J. (2016). Politische Vertrauenskrise? Die kommunikative Konstruktion von Politikervertrauen im lebensweltlichen Kontext. Wiesbaden: Springer.

Hopmann, D. N., Shehata, A., \& Strömbäck, J. (2015). Contagious media effects. How media use and exposure to game-framed news influence media trust. Mass Communication and Society, 18, 776-798.

Jackob, N. (2012). Gesehen, gelesen - geglaubt? Warum die Medien nicht die Wirklichkeit abbilden und die Menschen ihnen dennoch vertrauen. München: Olzog.

Jackob, N., Quiring, O., \& Schemer, C. (2017). Wölfe im Schafspelz? Warum manche Menschen denken, dass man Journalisten nicht vertrauen darf - und was das mit Verschwörungstheorien zu tun hat. In K. N. Renner, T. Schultz \& J. Wilke (Hrsg.), Journalismus zwischen Autonomie und Nutzwert (S. 225-249). Köln: Herbert von Halem.

Jackob, N., Jakobs, I., Quiring, O., Schultz, T., Schemer, C., \& Ziegele, M. (2019a). Medienskepsis und Medienzynismus - Funktionale und dysfunktionale Formen von Medienkritik. Communicatio Socialis, 52, 19-35.

Jackob, N., Schultz, T., Jakobs, I., Ziegele, M., Quiring, O., \& Schemer, C. (2019b). Medienvertrauen im Zeitalter der Polarisierung. Media Perspektiven, o.J.(5), 210-220.

Jakobs, I. (2018). Vertrauenszuschreibungen an Medien. Eine experimentelle Studie zu den Ebenen von Vertrauen in Medien. Dissertation. Mainz: Universität Mainz. 
Jakobs, I., Schultz, T., Viehmann, C., Quiring, O., Jackob, N., Ziegele, M., \& Schemer, C. (2021). Medienvertrauen in Krisenzeiten. Mainzer Langzeitstudie Medienvertrauen 2020. Media Perspektiven, o.J.(3), 152-162.

Jones, D. A. (2004). Why americans don't trust the media: a preliminary analysis. The International Journal of Press/Politics, 9, 60-75.

Kohring, M. (2004). Vertrauen in Journalismus. Theorie und Empirie. Konstanz: UVK.

Lee, T.-T. (2010). Why they don't trust the media: an examination of factors predicting trust. American Behavioral Scientist, 54, 8-21.

Luhmann, N. (1989). Vertrauen. Ein Mechanismus der Reduktion sozialer Komplexität. Stuttgart: Enke. zuerst 1968

Möhring, W., \& Schlütz, D. (2010). Die Befragung in der Medien- und Kommunikationswissenschaft. Eine praxisorientierte Einführung. Wiesbaden: VS.

Ognyanova, K. (2019). The social context of media trust: a network influence model. Journal of Communication, 69, 544-567.

Paternoster, R., Brame, R., Mazerolle, P., \& Piquero, A. (1998). Using the correct statistical test for the equality of regression coefficients. Criminology, 36, 859-866.

Peifer, J. T. (2018). Imitation as flattery: how TV news parody's media criticism can influence perceived news media importance and media trust. Journalism \& Mass Communication Quarterly, 95, 734-756.

Pingree, R.J., Quenette, A.M., Tchernev, J.M., \& Dickinson, T. (2013). Effects of media criticism on gatekeeping trust and implications for agenda setting. Journal of Communication, 63, 351-372.

Prochazka, F., \& Schweiger, W. (2019). How to measure generalized trust in news media? An adaptation and test of scales. Communication Methods and Measures, 13, 26-42.

Reinemann, C., Fawzi, N., \& Obermaier, M. (2017). Die „Vertrauenskrise“ der Medien - Fakt oder Fiktion? Zu Entwicklung, Stand und Ursachen des Medienvertrauens in Deutschland. In V. Lilienthal \& I. Neverla (Hrsg.), „Lügenpresse“. Anatomie eines politischen Kampfbegriffs (S. 77-94). Köln: Kiepenheuer und Witsch.

Schielicke, A.-M., Mothes, C., \& Donsbach, W. (2014). Vertrauen in Journalisten: Trends \& Einflussfaktoren. In B. Stark, O. Quiring \& N. Jackob (Hrsg.), Von der Gutenberg-Galaxis zur Google-Galaxis: Alte und neue Grenzvermessung nach 50 Jahren DGPuK (S. 247-269). Konstanz: UVK.

Schranz, M., Schneider, J., \& Eisenegger, M. (2016). Medienvertrauen - eine vergleichende Perspektive. In fög - Forschungsinstitut Öffentlichkeit und Gesellschaft Universität Zürich (Hrsg.), Qualität der Medien. Basel: Schwabe.

Schultz, T., Ziegele, M., Jakobs, I., Jackob, N., Quiring, O., \& Schemer, C. (2020). Medienzynismus weiterhin verbreitet, aber mehr Menschen widersprechen. Mainzer Langzeitstudie Medienvertrauen 2019. Media Perspektiven, o.J.(6), 322-330.

Siegert, G. (2001). Medien Marken Management. Relevanz, Spezifika und Implikationen einer medienökonomischen Profilierungsstrategie. München: Fischer.

Silberer, G. (2001). Medien als Marken. In R. Köhler, W. Majer \& H. Wiezorek (Hrsg.), Erfolgsfaktor Marke: neue Strategien des Markenmanagements (S. 237-252). München: Vahlen.

Strömbäck, J., Tsfati, Y., Boomgaarden, H., Damstra, A., Lindgren, E., Vliegenthart, R., \& Lindholm, T. (2020). News media trust and its impact on media use: toward a framework for future research. Annals of the International Communication Association, 44, 139-156.

Sztompka, P. (1999). Trust. A sociological theory. Cambridge: Cambridge University Press.

Tsfati, Y. (2003a). Media scepticism and climate of opinion perception. International Journal of Public Opinion Research, 15, 65-82.

Tsfati, Y. (2003b). Does audience skepticism of the media matter in agenda setting? Journal of Broadcasting \& Electronic Media, 47, 157-176.

Tsfati, Y., \& Ariely, G. (2014). Individual and contextual correlates of trust in media across 44 countries. Communication Research, 41, 760-782.

Tsfati, Y., \& Capella, J.N. (2003). Do people watch what they do not trust? Exploring the association between news media skepticism and exposure. Communication Research, 30, 504-529.

Tsfati, Y., \& Capella, J. N. (2005). Why do people watch news they do not trust? The need for cognition as a moderator in the association between news media scepticism and exposure. Media Psychology, 7 , 251-271.

Tsfati, Y., \& Peri, Y. (2006). Mainstream media scepticism and exposure to Sectorial and Extranational news media: the case of Israel. Mass Communication \& Society, 9, 165-187.

Yamamoto, M., Lee, T.-S., \& Ran, W. (2016). Media trust in a community context: a multilevel analysis of individual- and prefecture-level sources of media trust in Japan. Communication Research, 43, 131-154. 
Dr. Ilka Jakobs ist wissenschaftliche Mitarbeiterin und Leiterin des Studienbüros am Institut für Publizistik der Johannes Gutenberg-Universität Mainz.

Dr. N. Jackob ist Privatdozent und Geschäftsführer des Studienbüros am Institut für Publizistik der Johannes Gutenberg-Universität Mainz.

Dr. T. Schultz ist Professor am Journalistischen Seminar der Johannes Gutenberg-Universität Mainz.

Dr. M. Ziegele ist Junior-Professor und Leiter der Nachwuchsforschergruppe „Deliberative Discussions in the Social Web“ (DEDIS) am Institut für Sozialwissenschaften der Heinrich-Heine-Universität Düsseldorf.

Dr. C. Schemer ist Professor am Institut für Publizistik der Johannes Gutenberg-Universität Mainz.

Dr. O. Quiring ist Professor am Institut für Publizistik der Johannes Gutenberg-Universität Mainz. 